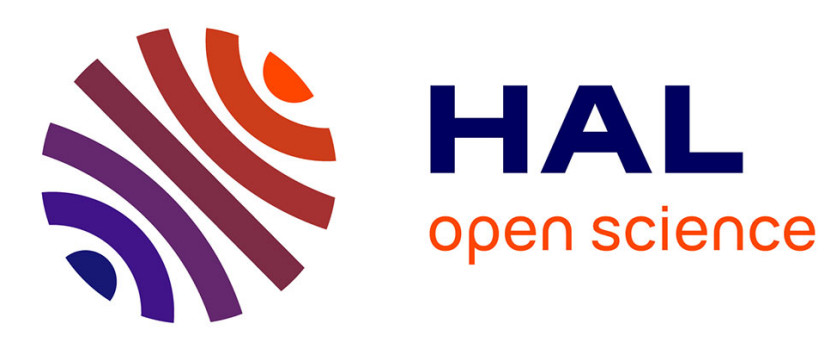

\title{
Attaques spéculatives et crédibilité du régime de change: quel arbitrage pour le policy-mix?
}

Jean-Pierre Allegret, Marie-Noëlle Calès

\section{To cite this version:}

Jean-Pierre Allegret, Marie-Noëlle Calès. Attaques spéculatives et crédibilité du régime de change: quel arbitrage pour le policy-mix?. Revue Economique, 2001, 52 (2), pp.249-264. 10.3917/reco.522.0249 . hal-01660196

\section{HAL Id: hal-01660196 https://hal.science/hal-01660196}

Submitted on 15 May 2018

HAL is a multi-disciplinary open access archive for the deposit and dissemination of scientific research documents, whether they are published or not. The documents may come from teaching and research institutions in France or abroad, or from public or private research centers.
L'archive ouverte pluridisciplinaire HAL, est destinée au dépôt et à la diffusion de documents scientifiques de niveau recherche, publiés ou non, émanant des établissements d'enseignement et de recherche français ou étrangers, des laboratoires publics ou privés. 


\title{
Attaques spéculatives et crédibilité du régime de change : quel arbitrage pour le policy-mix ?
}

\author{
Jean-Pierre Allegret* \\ Marie-Noëlle Calès*
}

\begin{abstract}
Les crises asiatique et brésilienne ont intensifié le débat relatif à la politique économique à mettre en xuvre par les autorités face à une crise de change. L'augmentation des taux d'intérêt accompagnée d'une politique budgétaire restrictive peut se révéler contreproductive eu égard à l'objectif de stabilisation du change. Ce papier analyse la question de la crédibilité du policy-mix mis en cuvre par les autorités pour contrer une crise de change. La perspective adoptée se situe dans la lignée des travaux développés en termes de crise de crédibilité mais elle s'en démarque en introduisant explicitement la politique budgétaire dans l'analyse. Le modèle suggère qu'une politique budgétaire restrictive n'est pas nécessairement la décision la plus crédible.
\end{abstract}

\section{SPECULATIVE ATTACKS AND CREDIBILITY OF EXCHANGE RATE REGIME : WHAT POLICY-MIX TRADE-OFF ?}

Since the Asian and Brazilian exchange rate crises have occurred, the reaction of authorities has been the subject of an intense debate. Indeed, coping with a speculative attack, domestic authorities both increase interest rates and contract fiscal policy. It appears that such a combination of policy instruments is counterproductive. This paper analyses the credibility of the policy-mix used by authorities. But, opposite to traditional credibility models, fiscal policy is explicitly introduced in the loss function of the authorities. The model suggests that a restrictive fiscal policy is not necessary a credible decision to defence the exchange rate target.

Classification JEL : E5 ; E6 ; F4

\section{INTRODUCTION}

Les crises de change se sont multipliées depuis la fin des années 80, affectant aussi bien les pays développés que les économies émergentes. En dépit de la diversité des situations économiques conduisant à ces crises, celles-ci ont en commun de révéler le caractère coûteux de la défense de l'objectif de change lorsqu'une attaque spéculative se produit. En effet, les autorités monétaires

*. Groupe d'Analyse et de Théorie Economique, UMR 5824 du CNRS et Université-Lumière Lyon 2, 93 chemin des Mouilles 69130 Ecully. E-mail : allegret@gate.cnrs.fr ; cales@gate.cnrs.fr 
réagissent habituellement à des tensions sur le change en augmentant les taux d'intérêt afin de décourager la spéculation. Or, cet accroissement des taux d'intérêt exerce une influence négative sur les variables macro-économiques domestiques, influence qui peut produire un cercle vicieux identifié dans la deuxième génération des modèles d'attaque spéculative (Obstfeld, [1994]). Les crises asiatique et brésilienne ont intensifié le débat relatif aux politiques économiques menées par les autorités face à une crise de change. Radelet - Sachs [1998] ont, entre autres, souligné le caractère improductif de l'augmentation des taux d'intérêt accompagnée d'une politique budgétaire restrictive. En effet, un tel policy-mix serait non crédible, aggravant alors la situation économique des pays dont les monnaies sont attaquées.

L'objectif de ce papier est précisément de focaliser l'analyse sur le policy-mix mis en œuvre pour contrer une crise de change en plaçant le dilemme inflation chômage (soit aussi le dilemme défense de la monnaie - activité domestique) au cœur du processus de décision des autorités. En effet, à notre sens, les crises de change ne se réduisent pas exclusivement à un problème de finances publiques. A la différence des modèles traditionnels où la variable budgétaire est principalement à l'origine de la crise, la politique budgétaire est ici envisagée comme un instrument de maîtrise des anticipations de dépréciation de la monnaie. La perspective adoptée se situe dans la lignée des travaux développés en termes de crises de crédibilité (Agénor et Masson, [1999] ; Bensaïd et Jeanne, [1997] ; Drazen et Masson, [1994]), mais elle s'en démarque en introduisant explicitement la politique budgétaire dans l'analyse. Il s'agit alors d'examiner la crédibilité du policy-mix choisi par les autorités pour défendre le taux de change et de montrer qu'une politique budgétaire restrictive - associée à une politique monétaire stricte - n'est pas toujours la décision la plus crédible.

La première section est ainsi consacrée à l'analyse des stratégies de politique économique déployées par les autorités de quelques pays émergents frappés par des crises monétaires et à leurs principales conséquences. Elle met l'accent sur le policymix mis en œuvre dans ces pays et souligne la difficulté de ces derniers à stabiliser les régimes de change. La deuxième section propose une modélisation des choix des autorités monétaires qui permet de mettre en évidence les conditions dans lesquelles la politique budgétaire peut contribuer à la stabilisation du taux de change. Enfin, dans la troisième section, les effets d'une règle budgétaire flexible sur la crédibilité de l'utilisation de l'arme budgétaire sont analysés.

\section{L'ENSEIGNEMENT DES CRISES MONETAIRES RECENTES}

Les crises de change confrontent les autorités des pays concernés au traditionnel problème du conflit d'objectifs. Ainsi, la plupart des économies émergentes frappées par les crises de change au cours des années 90 ont dû, à des degrés divers, défendre simultanément deux objectifs difficilement compatibles : d'une part, restaurer la croissance économique, notamment par une politique de soutien au secteur financier, et, d'autre part, stabiliser le taux de change. Dans un monde intégré financièrement, ces deux objectifs sont liés par la confiance des investisseurs. Plus précisément, une contraction trop forte de l'autorité et/ou une dépréciation importante de la monnaie peuvent conduire à un processus cumulatif de crise monétaire par la perte de 
confiance des investisseurs (Furman et Stiglitz, [1998]).

\section{LES PROGRAMMES MIS EN CEUVRE PAR LE FMI}

Afin d'effectuer un arbitrage entre stabilisation interne et stabilisation du change, les autorités disposent de deux instruments : la politique monétaire et la politique budgétaire. Or, les pays qui ont été soumis aux programmes de stabilisation du Fonds Monétaire International, soit à la suite des crises de change - Corée du Sud, Indonésie, Russie et Thaïlande - soit préventivement (Brésil), ont utilisé un policymix similaire : contraction monétaire et ajustement budgétaire.

Les programmes mis en œuvre par le FMI en Thaïlande (août 1997), en Indonésie (novembre 1997) et en Corée du Sud (décembre 1997), reposent sur le diagnostic selon lequel ces économies sont frappées par une crise de liquidité. Il s'est donc agi de mobiliser les instruments de la politique économique de manière à stopper l'hémorragie des capitaux et à limiter dans le même temps le besoin de capitaux extérieurs. En revanche, malgré d'importantes difficultés bancaires en Russie et au Brésil, les programmes de stabilisation du FMI à la suite des crises du rouble (1998) et du real (1999) semblent davantage marqués par des déséquilibres macroéconomiques traditionnels. Cependant, il convient de noter que ces programmes sont très proches de ceux initiés en Asie dans la mesure où, outre la contraction monétaire destinée à stabiliser les taux de change des deux monnaies, les orientations budgétaires ont été elles aussi marquées par le souci de réaliser un ajustement. Au Brésil comme en Russie, les experts du FMI interprètent les difficultés des autorités à assainir le budget comme un facteur fragilisant l'accrochage du real ${ }^{1}$ ou du rouble au dollar.

\section{LE MANQUE DE CREDIBILITE DES INTERVENTIONS DU FMI}

L'annexe 1 qui présente un certain nombre d'indicateurs macroéconomiques pour les pays sous programme de stabilisation du FMI montre que, conformément aux lignes de défense traditionnelles des autorités monétaires, l'ensemble des pays ont non seulement recouru à des ventes de devises pour soutenir leur monnaie, mais qu'ils ont également augmenté massivement les taux d'intérêt à court terme au moment où l'attaque spéculative s'est produite. Dans cinq des neufs épisodes considérés - Mexique, Brésil (1997), Corée du Sud, Indonésie et Thaïlande (1997) les taux d'intérêt à court terme n'ont pas retrouvé leur niveau d'avant la crise deux trimestres après. Ces tensions monétaires se sont accompagnées de reculs de la production dans tous les pays ayant subi une attaque. Dans sept épisodes sur neuf Mexique, Argentine, Brésil (1997), Corée du Sud, Thaïlande (1997 et 1998) et

1. L'opposition du Congrès aux plans d'ajustement du gouvernement a contribué à fragiliser cet accrochage. Les difficultés du gouvernement brésilien à assainir le budget sont également liées à la structure fédérale du pays, les états composant la fédération subissant eux aussi une crise budgétaire importante. 
Russie - les niveaux de production deux trimestres après la crise demeurent inférieurs à ceux observés un trimestre avant.

Or, en dépit du durcissement de la politique monétaire, les attaques sur les monnaies ont réussi. En effet, à l'exception de l'Argentine dotée d'un currency board, les monnaies des pays considérés ont subi des dépréciations parfois massives sans stabilisation apparente le trimestre suivant l'attaque. Les difficultés rencontrées par les autorités monétaires du Brésil (1997 et 1999), d’Indonésie, de Russie et de Thaïlande (1997 et 1998) à stabiliser leur monnaie au cours des trimestres suivant la crise - et ce, alors même qu'ils étaient sous programme du FMI - suggèrent que les réactions de politique monétaire n'ont pas été jugées crédibles par les agents privés. Alors même que ces programmes reposaient sur la volonté de réaliser une stabilisation budgétaire, tous les pays sous programme FMI (à l'exception de la Russie) ont subi une dégradation des soldes budgétaires au cours des trimestres suivant la crise de change.

Ainsi, en sacrifiant l'objectif interne de croissance au profit de l'objectif externe de stabilisation du taux de change, les réactions des autorités nationales et internationales ont été confrontées à un problème de crédibilité. Radelet et Sachs [1998] suggèrent même que les programmes du FMI - exigeant de prime abord à la fois la contraction monétaire et budgétaire - ont pu aggraver la situation et empêcher par là-même la stabilisation de l'économie. Ils observent que chaque annonce de programme a été suivie d'une accélération de la dépréciation de la monnaie domestique. En d'autres termes, un conflit entre équilibres interne et externe est clairement apparu, suggérant que la stabilisation monétaire pouvait être non crédible car intenable sur le plan domestique.

Le Brésil apparaît de ce point de vue être un cas d'école. En effet, dans un contexte de tensions sur le taux de change du real, le FMI est intervenu préventivement. Cette intervention n'a pas empêché le décrochage du real contre dollar début 1999. Selon le FMI, ce décrochage a été lié à l'incertitude politique régnant au Brésil concernant le degré d'engagement à réaliser l'ajustement budgétaire requis. Cependant, la politique monétaire restrictive a engendré une importante contraction de l'activité qui, par le jeu des stabilisateurs automatiques, a aggravé le déficit budgétaire du pays. Ainsi, les autorités brésiliennes semblent avoir été prises dans un cercle vicieux : la stabilité des changes appelle un ajustement budgétaire, mais celui-ci est rendu non soutenable étant donné la politique monétaire restrictive, d'où une accentuation de l'instabilité ${ }^{2}$. Il semblerait donc que les autorités ne soient généralement pas en mesure de réaliser l'ajustement budgétaire exigé par le FMI. Dans cette perspective, n'est il pas alors plus opportun d'agir sur la variable budgétaire plutôt que d'en subir les évolutions?

L'analyse des plans de stabilisation du FMI pose ainsi la question du policy-mix mis en œuvre et la question de sa crédibilité. De nombreux auteurs se sont interrogés sur la capacité d'une hausse des taux d'intérêt à stabiliser le taux de change. Les résultats des études de Furman et Stiglitz [1998], de Kraay [1998] et de Goldfajn et Gupta [1999] suggèrent que le durcissement de la politique monétaire - via les taux

2. Ce qui fait dire à Sachs [1999] que c'est finalement le FMI qui a provoqué la crise du real par une politique non soutenable sur le plan interne. 
d'intérêt - est loin d'être nécessairement la réponse appropriée ${ }^{3}$. Goldfajn et Gupta [1999], Furman et Stiglitz [1998] mettent en avant l'importance de la situation économique de départ et des institutions politique et/ou économiques pour appréhender l'efficacité de la défense du régime de change ${ }^{4}$.

Devant ces conclusions, ne peut-on pas envisager que, dès lors que le pays dispose de marges de manœuvre budgétaires, il peut être opportun de les utiliser pour faciliter la défense du taux de change? Une politique budgétaire moins restrictive, laissant jouer les stabilisateurs automatiques pourrait ainsi contribuer à rendre plus crédible une politique monétaire stricte visant à défendre le taux de change. Ainsi l'arme budgétaire peut être utilisée pour accroître le degré de résistance des autorités aux anticipations de dépréciation de la monnaie. Le recours à l'instrument budgétaire afin de limiter les effets récessionnistes des politiques de contraction monétaire repose toutefois sur un préalable important: l'existence de marges de manœuvre budgétaires. Dans le cas contraire, c'est à un autre problème de crédibilité que les autorités seront confrontées.

Le modèle développé dans la partie suivante place précisément l'arbitrage entre l'augmentation des dépenses publiques et la dépréciation monétaire au cœur du processus de décision des autorités.

\section{LE ROLE DE LA POLITIQUE BUDGETAIRE DANS LA DEFENSE DU REGIME DE CHANGE}

Les variables budgétaires occupent une place privilégiée dans les modèles d'attaque spéculative. Les modèles de la première génération considèrent ainsi que les déficits budgétaires constituent un déséquilibre fondamental au sens où ils peuvent précipiter une crise de change (Krugman [1979]). Du point de vue du policy-mix lié aux crises de change, une limite importante des modèles précédents est de ne pas considérer l'interdépendance entre le coût de la défense du régime de change et l'évolution de la situation budgétaire. Dans cette perspective, les modèles de la seconde génération nous semblent davantage adaptés pour analyser une telle question. Obstfeld [1994] et De Grauwe, [1997] introduisent le mécanisme de la taxe inflationniste pour montrer pourquoi le niveau de la dette publique peut inciter le gouvernement à dévaluer. Ces modèles suggèrent que la prise en compte des coûts de défense du régime de change peut limiter les marges de manœuvre des autorités afin de maintenir simultanément l'équilibre interne (emploi) et l'équilibre externe

3 Basurto et Ghosh [2000] contestent ces résultats en considérant que le taux d'intérêt n'est pas un bon indicateur des tensions monétaires. Ils utilisent à cette fin l'offre de monnaie pour montrer que le durcissement monétaire conduit en général à l'effet attendu (une appréciation de la monnaie domestique). Cependant, ce résultat est obtenu à partir d'un modèle monétaire de détermination des taux de change dont on connaît les limites.

4 Goldfajn et Gupta montrent que la fragilité bancaire affaiblit l'efficacité de la politique monétaire pour stabiliser le taux de change. Furman et Stiglitz soulignent que la réaction de politique monétaire à la suite d'une attaque est d'autant plus efficace que le pays est inflationniste (cas du Brésil), mais elle l'est d'autant moins que le taux d'inflation est faible et que l'approfondissement financier est important (cas de l'Indonésie). 
(taux de change). Surtout, ils conduisent à penser que la politique budgétaire - saisie le plus fréquemment à travers la dette publique - peut engendrer une attaque spéculative. Ce résultat légitime les politiques qui visent à réduire les déficits budgétaires ou à limiter l'ampleur de l'utilisation de l'arme budgétaire à des fins de stabilisation. Or, les conclusions issues des travaux empiriques apparaissent partiellement en contradiction avec les résultats théoriques, suggérant que les autorités peuvent, dans certaines conditions, jouer sur la politique budgétaire pour créer des marges de manœuvre utiles à la défense du taux de change (Kaminsky, Lizondo et Reinhart, [1997] ; Sachs, Tornell et Velasco, [1996]). Autrement dit, mener en parallèle une politique monétaire stricte et une politique budgétaire plus souple serait dans certains cas un policy-mix plus crédible qu'une politique doublement restrictive. L'objectif de cette partie est de montrer que la politique budgétaire, loin de constituer nécessairement un déterminant prépondérant d'une attaque spéculative, peut au contraire se concevoir comme un élément de la stratégie de défense du régime de change.

\section{UN MODELE DE CREDIBILITE AVEC ARBITRAGE TAUX DE CHANGE / PRODUCTION / DEPENSES PUBLIQUES}

Le comportement des autorités est modélisé à partir d'une approche en termes de crédibilité et se focalise sur l'arbitrage taux de change, production et dépenses publiques. L'approche qui est retenue dans ce modèle s'inspire des modèles de crédibilité développés par Bensaid et Jeanne [1997] et par Drazen et Masson [1994] qui placent l'arbitrage inflation/chômage au cœur de la mécanique du taux de change. A la différence de ces travaux, la politique budgétaire et les préoccupations des autorités en matière de finances publiques sont intégrées dans le modèle, ce qui se traduit par une fonction de perte élargie incluant la variable budgétaire. La fonction d'offre est une courbe de Phillips augmentée des anticipations: toute inflation (ou toute dépréciation) non anticipée permet d'augmenter le PIB. Par ailleurs, il est fait l'hypothèse, ainsi que le fait Artus [1995], que les dépenses publiques ont une influence, même partielle, sur le niveau de production. Les autorités adoptent un comportement optimisateur sous une contrainte d'équilibre des finances publiques, mais le problème ne peut se résumer, ainsi que l'envisagent Radelet et Sachs [1998] et d'Obstfeld [1994], à un simple arbitrage entre taux de change et taux d'imposition. Il est ainsi plus pertinent d'envisager un arbitrage taux de change/production/dépenses publiques.

Le modèle de crédibilité présenté dans ce texte prend en compte la spécificité des économies asiatiques. Il permet en particulier d'interpréter les conséquences budgétaires des crises bancaires. Selon Burnside, Eichenbaum et Rebelo [1998], la présence de la garantie implicite de l'Etat envers les intermédiaires financiers pèse sur les déficits publics futurs. Cette garantie conduit nécessairement les agents privés à anticiper une monétisation de la dette publique incompatible avec le maintien du régime de change. Les autorités doivent donc dès aujourd'hui réduire les déficits budgétaires ou accroître leurs excédents. La perspective adoptée ici est différente. En effet, la réduction dès aujourd'hui des déficits budgétaires peut à l'inverse de l'effet souhaité par les autorités provoquer une dégradation des anticipations de dépréciation. Plus précisément, la contraction budgétaire 
s'accompagne d'une récession qui aggrave la situation des banques. Le rationnement du crédit qui en résulte entraîne une contraction de l'activité. Les moindres recettes fiscales dégradent alors la situation budgétaire du pays et renforcent les anticipations de dépréciation.

Les principales hypothèses retenues dans le modèle se résument donc ainsi : (i) la fonction de perte des autorités est quadratique et comprend trois objectifs, le taux de change $\left(\mathrm{e}_{\mathrm{t}}\right)$, la croissance $\left(\mathrm{y}_{\mathrm{t}}\right)$ et les dépenses publiques $\left(\mathrm{g}_{\mathrm{t}}\right)$; (ii) les autorités - à savoir ici le gouvernement étant donné l'absence d'indépendance de fait des banques centrales dans la plupart des économies émergentes - se préoccupent de l'équilibre des finances publiques $\left(\mathrm{g}_{\mathrm{t}}\right)$, mais elles ont une aversion à l'augmentation des impôts $\left(\tau_{t}=\bar{\tau}\right)$; (iii) la fonction d'offre est une courbe à la Lucas ; (iv) les dépenses publiques ont un effet partiel sur le niveau de production (mesuré par $\delta$ ) ; (v) les agents privés réalisent des anticipations rationnelles. Ces hypothèses se traduisent dans les quatre équations suivantes :

$$
\begin{aligned}
& L_{t}=\frac{a}{2} \pi_{t}^{2}+\frac{b}{2}\left(y_{t}-y^{*}\right)^{2}+\frac{1}{2} g_{t}^{2} \\
& y_{t}=\pi_{t}-\pi_{t}^{e}+\delta g_{t}-\bar{\tau} \\
& g_{t}+d_{t-1}\left(1+i_{t}-\pi_{t}\right)=d_{t}+\pi_{t}+\bar{\tau} \\
& \pi_{t}=e_{t}-e_{t-1}, \text { selon l'hypothèse de parité des pouvoirs d'achat. }
\end{aligned}
$$

A chaque période les autorités doivent déterminer $\alpha_{t}$ qui désigne la norme de progression de la dette à la période $\mathrm{t}$. La dette à la période $\mathrm{t}$ peut alors s'écrire $d_{t}=\alpha_{t} d_{t-1}$, soit $d_{t}=\alpha_{t} d$. En effet, la dette de la période t-1 est considérée comme remboursée à la période suivante et constitue une dépense de la période $\mathrm{t}$, donc $d_{t-1}=d$. Les autorités choisissent alors la dette de la période $\mathrm{t}$ en vue de rembourser la dette de la période précédente mais également afin de financer les dépenses publiques courantes. Par ailleurs, le taux d'intérêt nominal inclut des anticipations d'inflation, ce qui se traduit par $i_{t}=r_{t}+\pi_{t}^{e}$. La contrainte budgétaire se réécrit ainsi :

$$
g_{t}+d\left(1+r_{t}-\pi_{t}+\pi_{t}^{e}\right)=\alpha_{t} d+\pi_{t}+\bar{\tau}
$$

Les autorités monétaires doivent donc choisir à chaque période la norme de progression de la dette de même qu'elles doivent déterminer l'évolution du taux de change, c'est-à-dire celle de l'inflation compte tenu de l'hypothèse de parité des pouvoirs d'achat. L'objectif est alors de déterminer le comportement optimal des autorités en matière de taux de change. Trois cas seront envisagés.

En changes flexibles (cas $n^{\circ} 1$ ), les agents anticipent que les autorités vont mettre en œuvre une politique discrétionnaire de dépréciation du taux de change. Ainsi les autorités cherchent à minimiser leur fonction de perte (1) sous les contraintes (2), (4) et (5), par rapport à $\alpha_{t}$ et $\pi_{\mathrm{t}}$. Après application des anticipations rationnelles sur les conditions de premier ordre, on obtient les anticipations d'inflation et de progression de la dette publique, dont on déduit ensuite l'inflation et la croissance de la dette 
publique réalisées.

Lorsque les autorités s'engagent à défendre le taux de change (cas n ${ }^{\circ} 2$ ), cela se traduit par la mise en ouvre d'une règle monétaire : $\pi_{t}=0$. Ainsi les autorités cherchent à minimiser leur fonction de perte $\mathrm{E}[\mathrm{L}]$ sous les contraintes (2), (4) et (5), par rapport aux variables anticipées $\pi_{t}^{e}$ et $\alpha_{t}^{e}$. Il faut, dans un premier temps, montrer que la défense du taux de change est une décision optimale, c'est à dire que, du point de vue des autorités, maintenir $\pi_{t}=0$ est optimal. Pour cela, nous posons la règle $\pi_{t}=K$, ce qui conduit à minimiser la fonction de perte rapport à $\mathrm{K}$ et $\alpha_{t}^{e}$.

On considère enfin un dernier cas ( $\operatorname{cas~}^{\circ} 3$ ), dans lequel un choc d'offre négatif se produit à la suite de l'augmentation des taux d'intérêt consécutive à une attaque spéculative sur la monnaie domestique. Le modèle est modifié par l'introduction d'un choc dans la fonction d'offre qui devient alors :

$$
y_{t}=\pi_{t}-\pi_{t}^{e}+\delta g_{t}-\bar{\tau}+\varepsilon_{t}
$$

On peut supposer que les autorités ont une information privilégiée sur les modifications des taux d'intérêt et sur le moment où peuvent se produire des chocs d'offre. Elles peuvent provoquer des chocs d'offre surprise et tenter de surprendre, au moins partiellement, les agents. Pour déterminer les marges de manœuvre qui s'offrent aux autorités, il convient de déterminer au préalable la règle de change optimale :

$$
\pi_{t}=\mu+\vartheta \varepsilon_{t}
$$

Le programme consiste alors à minimiser la fonction de perte anticipée, par rapport à $\vartheta$, $\mu$ et $\alpha_{t}$ sous les conditions (5), (6) et (7).

\section{DES CHANGES FLEXIBLES AUX REGLES DE TAUX DE CHANGE : QUELLES CONSEQUENCES POUR LA POLITIQUE BUDGETAIRE ?}

En changes flexibles (cas $n^{\circ} 1$ ), les autorités mènent une politique discrétionnaire. Il est optimal pour les autorités de réaliser les anticipations d'inflation, c'est à dire les anticipations de dépréciation des agents. Il est également optimal de financer la dépense publique par la taxe inflationniste et par l'augmentation de la dette publique. Si $\alpha_{t}^{e}>1$ (réciproquement si $\alpha_{t}^{e}<1$ ), alors les agents anticipent une progression de la dette publique (réciproquement une baisse de la dette). La condition $\alpha_{t}^{e}>1$ est remplie si on vérifie :

$$
d \in\left[\frac{\bar{\tau}}{r}+\frac{b\left(y^{*}+\bar{\tau}\right)(1-a \delta)}{r a\left(b \delta^{2}+1\right)}, \bar{d}\right], \text { où } \bar{d} \text { est le seuil au- }
$$

delà duquel le gouvernement a une aversion à l'augmentation de la dette.

Si d appartient à cet intervalle, les agents anticipent une progression de la dette 
publique. Au delà d'un certain seuil, les autorités sont incitées à augmenter la dette de période en période, dans la mesure où la contrainte de solvabilité $(\bar{d})$ n'est pas encore atteinte. L'impact sur la production est en effet d'autant plus fort que la dette est initialement élevée. C'est par ailleurs clairement la défense par les autorités d'un objectif de production $\mathrm{y}^{*}$ qui provoque à la fois des anticipations de dépréciation et d'augmentation des dépenses publiques : en effet, $\pi_{t}^{e}$ et $\alpha_{t}^{e}$ dépendent tous les deux de $\mathrm{y}^{*}$. Ceci souligne l'importance de l'arbitrage inflation-production dans un processus de dépréciation monétaire.

\begin{tabular}{|c|c|c|c|}
\hline & & INFLATION & $\begin{array}{l}\text { CROISSANCE DE LA } \\
\text { DETTE PUBLIQUE }\end{array}$ \\
\hline \multirow{2}{*}{$\begin{array}{l}\text { CAS N }{ }^{\circ} 1: \\
\text { LES CHANGES } \\
\text { FLEXIBLES }\end{array}$} & 苋 & $\pi_{t}^{e}=\frac{b\left(y^{*}+\bar{\tau}\right)}{a\left(b \delta^{2}+1\right)}$ & $\begin{array}{l}\alpha_{t}^{e}=\frac{\left(y^{*}+\bar{\tau}\right) b(a \delta-1)}{a d\left(b \delta^{2}+1\right)} \\
-\frac{\bar{\tau}}{d}+(1+r)\end{array}$ \\
\hline & 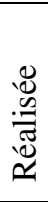 & $\pi_{t}=\frac{b\left(y^{*}+\bar{\tau}\right)}{a\left(b \delta^{2}+1\right)}$ & $\begin{array}{l}\alpha_{t}=\frac{\left(y^{*}+\bar{\tau}\right) b(a \delta-1)}{a d\left(b \delta^{2}+1\right)} \\
-\frac{\bar{\tau}}{d}+(1+r)\end{array}$ \\
\hline \multirow{2}{*}{$\begin{array}{l}\text { CAS N } 2 \text { : } \\
\text { LA REGLE DE } \\
\text { TAUX DE } \\
\text { CHANGE }\end{array}$} & 苋 & $\pi_{t}^{e}=K=0$ & $\begin{array}{l}\alpha_{t}^{e}=\frac{b \delta\left(y^{*}+\bar{\tau}\right)}{d\left(b \delta^{2}+1\right)} \\
-\frac{\bar{\tau}}{d}+1+r\end{array}$ \\
\hline & 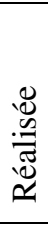 & $\pi_{t}=0$ & $\begin{array}{l}\alpha_{t}=\frac{b \delta\left(y^{*}+\bar{\tau}\right)}{d\left(b \delta^{2}+1\right)} \\
-\frac{\bar{\tau}}{d}+1+r\end{array}$ \\
\hline \multirow{2}{*}{$\begin{array}{l}\text { CAS N }{ }^{\circ}: \\
\text { CHOC D'OFFRE } \\
\text { ET REGLE DE } \\
\text { CHANGE }\end{array}$} & 苋 & $\pi_{t}^{e}=0$ & $\begin{array}{l}\alpha_{t}^{e}=\frac{b \delta\left(y^{*}+\bar{\tau}\right)}{d\left(b \delta^{2}+1\right)} \\
-\frac{\bar{\tau}}{d}+1+r\end{array}$ \\
\hline & 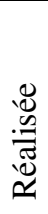 & $\pi_{t}=\frac{-b \varepsilon}{a+\left(b \delta^{2}+1\right)(1+d)^{2}+b}$ & $\begin{array}{l}\alpha_{t}=\frac{b \delta\left(y^{*}+\bar{\tau}\right)}{d\left(b \delta^{2}+1\right)} \\
-\frac{\bar{\tau}}{d}+1+r\end{array}$ \\
\hline
\end{tabular}

Dans une économie avec règle de change (cas $\left.n^{\circ} 2\right)$, il apparaît que la croissance anticipée de la dette publique est supérieure à celle d'une économie sans règle de change (cas $\left.n^{\circ} 1\right)$. Cela signifie que, du point de vue des autorités, il est optimal de reporter une partie du financement des dépenses publiques sur la dette dans la mesure où elles renoncent à la taxe inflationniste. Cependant, une telle règle de change est-elle crédible ? Les agents privés croiront en l'engagement des autorités dans la mesure où ils considèrent que les autorités disposent de marges de 
manœuvre budgétaire pour stimuler l'activité. Ainsi, $\alpha_{t}^{e}>1$, si la condition (9) est vérifiée :

$$
d \in\left[\frac{\bar{\tau}}{r}-\frac{b \delta\left(y^{*}+\bar{\tau}\right)}{r\left(b \delta^{2}+1\right)}, \bar{d}\right]
$$

La borne inférieure de la condition (9) est plus petite que celle de la condition (8). Cela signifie que, dans un régime avec règle de change, le seuil à partir duquel les autorités sont disposées à accroître les dépenses publiques est plus faible. Ainsi, le renoncement à la taxe inflationniste implique que l'efficacité des dépenses publiques apparaît plus rapidement. En réalité, c'est la crédibilité du policy-mix (règle de taux de change/croissance des dépenses publiques) qui est en jeu. La crédibilité de l'une ne peut pas être considérée sans la crédibilité de l'autre : la crédibilité de la règle de change s'appuie sur la crédibilité de la politique budgétaire mise en œuvre et vice-versa. Si les marges de manœuvre en matière budgétaire ne sont pas utilisées, il est vraisemblable que la défense de la règle ne paraisse pas crédible aux yeux des agents.

Le résultat principal du modèle avec choc d'offre (cas $n^{\circ} 3$ ) montre qu'il est optimal pour les autorités de procéder à la fois à une dépréciation ordonnée (de faible ampleur) de la monnaie domestique et à une augmentation de la dette publique. La possibilité d'obtenir une dépréciation ordonnée réside dans la capacité des autorités à mobiliser la politique budgétaire lorsqu'il apparaît un choc d'offre négatif. En effet, dans la mesure où l'augmentation de la dette publique respecte la condition (9), la réaction des autorités est crédible sur le marché des changes. En revanche, dans le cas où les autorités ne disposent d'aucune marge de manœuvre, alors la réaction budgétaire devient non crédible: aucune dépréciation ordonnée n'est possible. Il convient de se demander si les autorités peuvent obtenir une dépréciation ordonnée sans recourir à la politique budgétaire. Une telle stratégie nous semble non crédible étant donné que, dans ce cas, le coût de défense de la règle de change deviendrait exorbitant pour les autorités au cours des périodes suivantes.

L'utilisation de la politique budgétaire lors des crises de change est complexe à plus d'un titre. Tout d'abord, elle se heurte au traditionnel problème de la flexibilité de l'instrument. Cependant, le modèle précédent repose moins sur les stabilisateurs automatiques que sur l'impact des décisions budgétaires sur les anticipations des agents privés. Ainsi, le fait de ne pas réduire les dépenses budgétaires aujourd'hui, voire même de les augmenter, engendre un effet signal positif : l'anticipation de dépréciation de la monnaie domestique diminue grâce au renforcement de la capacité de défense des autorités dans le futur. Ensuite, si les autorités renoncent à durcir la politique budgétaire qui deviendrait alors pro-cyclique, elles se heurtent à une double difficulté. Les autorités doivent préalablement disposer de marges de manœuvre leur permettant de répondre de manière contra-cyclique aux effets négatifs de l'augmentation des taux d'intérêt. Un deuxième problème apparaît alors : celui de l'estimation par les autorités et par les agents privés de ces marges de manœuvre. Ce problème fait écho au fait que la politique budgétaire a une composante intertemporelle très importante liée notamment aux charges de la dette. Les anticipations des agents privés à propos des intentions à venir du gouvernement sont ici susceptibles de jouer un rôle décisif dans l'efficacité de la réaction de politique budgétaire. Ainsi, si les agents anticipent que les déficits courants - liés 
aux stabilisateurs automatiques - ne seront pas corrigés par des excédents futurs lorsque le cycle se sera retourné, alors l'effet immédiat de la politique budgétaire peut être contreproductif en raison de l'anticipation de la monétisation des déficits contemporains et futurs ${ }^{5}$. Il convient dans cette perspective de se demander si la prise en compte d'une règle budgétaire flexible permet de crédibiliser le policy-mix résultant d'une attaque spéculative.

\section{LA CREDIBILITE DU POLICY-MIX}

On suppose à nouveau que les autorités possèdent une information privilégiée sur les chocs d'offre. Elles peuvent ainsi jouer sur des effets de surprise en ce qui concerne les dépenses publiques. On introduit ici une règle budgétaire flexible, c'est-à-dire une règle contingente de l'état de l'économie. Elle doit permettre d'accroître les dépenses publiques en cas de choc négatif affectant la production sans engendrer d'anticipations inflationnistes significatives. Deux cas sont envisagés : d'une part, la présence d'une règle budgétaire souple dans un régime de changes fixes (cas $\left.n^{\circ} 4\right)$; d'autre part, un régime de double règle flexible (cas $n^{\circ} 5$ ). Des simulations numériques permettent ensuite d'affiner l'analyse des résultats.

\section{LE ROLE DES REGLES BUDGETAIRES FLEXIBLES}

Pour déterminer le policy-mix optimal des autorités dans le cas $\mathrm{n}^{\circ} 4$, on introduit une règle budgétaire souple prenant la forme suivante :

$$
\alpha_{t}=\mu+\vartheta \varepsilon_{t}
$$

Le programme consiste alors à minimiser la fonction de perte anticipée, par rapport à $K, \vartheta$ et $\mu$ sous les conditions (5), (6), (10) et $\pi_{t}=K$.

Le dernier régime (cas $\mathrm{n}^{\circ}$ ) est caractérisé par un abandon ponctuel des changes fixes parallèlement à la mise en œuvre d'une politique budgétaire contingente des états de l'économie. Cet abandon peut être modélisé par l'introduction d'une règle monétaire flexible :

$$
\pi_{t}=\gamma+\rho \varepsilon_{t}
$$

Les autorités minimisent alors la fonction de perte anticipée par rapport à

5 Ceci est d'autant plus vrai qu'on observe une corrélation négative entre le solde budgétaire et le rendement des obligations. Brady, Gavin et Haussmann [1998] estiment qu'à un solde budgétaire équilibré est associé un taux de rendement de $12 \%$ alors qu'à un déficit de l'ordre de $6 \%$ correspond un taux de $16 \%$. 
$\gamma, \rho, \vartheta$ et $\mu$ sous les conditions (5), (10) et (11).

\begin{tabular}{|c|c|c|c|}
\hline & & INFLATION & $\begin{array}{c}\text { CROISSANCE DE LA DETTE } \\
\text { PUBLIQUE }\end{array}$ \\
\hline \multirow{2}{*}{$\begin{array}{c}\text { CAS N }{ }^{\circ}: \\
\text { CHANGES FIXES } \\
\text { ET REGLE } \\
\text { BUDGETAIRE } \\
\text { SOUPLE }\end{array}$} & 兽 & $\pi_{t}^{e}=0$ & $\begin{array}{l}\alpha_{t}^{e}=\frac{b \delta\left(y^{*}+\bar{\tau}\right)}{d\left(b \delta^{2}+1\right)} \\
-\frac{\bar{\tau}}{d}+1+r\end{array}$ \\
\hline & 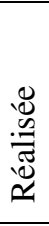 & $\pi_{t}=0$ & $\begin{array}{l}\alpha_{t}=\frac{b \delta\left(y^{*}+\bar{\tau}\right)}{d\left(b \delta^{2}+1\right)}-\frac{\bar{\tau}}{d} \\
+1+r-\frac{b \delta}{d\left(b \delta^{2}+1\right)} \varepsilon\end{array}$ \\
\hline \multirow{2}{*}{$\begin{array}{l}\text { CAS N }{ }^{\circ} \text { : } \\
\text { LE REGIME DE } \\
\text { DOUBLE REGLE } \\
\text { FLEXIBLE }\end{array}$} & 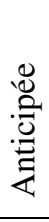 & $\pi_{t}^{e}=0$ & $\begin{array}{l}\alpha_{t}^{e}=\frac{b \delta\left(y^{*}+\bar{\tau}\right)}{d\left(b \delta^{2}+1\right)} \\
-\frac{\bar{\tau}}{d}+1+r\end{array}$ \\
\hline & 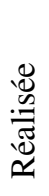 & $\pi_{t}=\frac{-b(1+\delta(1+d))}{a+b(1+\delta(1+d))} \varepsilon$ & $\begin{array}{l}\alpha_{t}=\frac{b \delta\left(y^{*}+\bar{\tau}\right)}{d\left(b \delta^{2}+1\right)}-\frac{\bar{\tau}}{d}+1+r \\
+\frac{b(d+1)(1+\delta(1+d))}{a d+b d(1+\delta(1+d))} \varepsilon\end{array}$ \\
\hline
\end{tabular}

Lorsqu'une règle budgétaire souple est utilisée parallèlement aux changes fixes (cas $\left.\mathrm{n}^{\circ} 4\right)$, il existe un biais à l'accroissement des dépenses publiques liées à la structure de l'économie (en particulier le niveau de l'impôt et l'objectif de production), comme dans le cas du régime de changes fixes sans règle budgétaire (cas $\mathrm{n}^{\circ} 1$ ), Cependant, lorsque l'on introduit une règle budgétaire souple, les dépenses publiques s'accroissent aussi sous l'effet d'un choc négatif affectant la production (dernier terme de droite de $\alpha_{t}$ ). On observe ainsi une absorption partielle du choc d'offre par le biais de la progression de la dette publique. Cette absorption renforce la crédibilité du régime de changes fixes dans la mesure où les autorités ne sont pas contraintes de sacrifier l'objectif de change en cas de choc d'offre négatif. La présence de la règle budgétaire souple joue le rôle de stabilisateur automatique.

Dans le cas de la double règle flexible (cas $\left.n^{\circ} 5\right)$, le premier constat qui s'impose concerne l'apparition d'une inflation positive qui traduit l'abandon ponctuel des changes fixes. Cette inflation positive permet une absorption partielle du choc d'offre négatif, de nature à limiter les anticipations de dépréciation des périodes suivantes. Par rapport au régime de changes fixes, on observe que, même si les anticipations des agents concernant les dépenses publiques sont inchangées, les dépenses publiques progressent moins vite. Il semblerait alors que les autorités aient tendance à reporter la compensation du choc d'offre sur l'inflation et pas sur les dépenses publiques. Les simulations effectuées confirment cette intuition. 


\section{LES PRINCIPAUX RESULTATS DES SIMULATIONS}

Des simulations numériques peuvent être effectuées afin d'observer la sensibilité des résultats, d'une part, aux chocs d'offre négatifs et, d'autre part, à la structure de l'économie (en particulier la préférences des autorités (b) et l'impact des dépenses publiques sur la production $(\delta)$ ). Afin d'étalonner le modèle, des valeurs plausibles ont été retenues pour les différents paramètres : $d=0,50 ; \tau=0,30 ; r=0,10 ; y^{*}=1$. Par ailleurs, $a=1-b$, dans la mesure où la fonction de préférence est exprimée en termes relatifs $\left(\frac{a}{2}+\frac{b}{2}+\frac{1}{2}=1\right)$.

La figure 1 représente les simulations effectuées pour les dépenses publiques en régime de changes fixes avec règle budgétaire souple en considérant deux chocs d'offre négatifs $(-0,1$ et $-0,01)$.

Figure 1
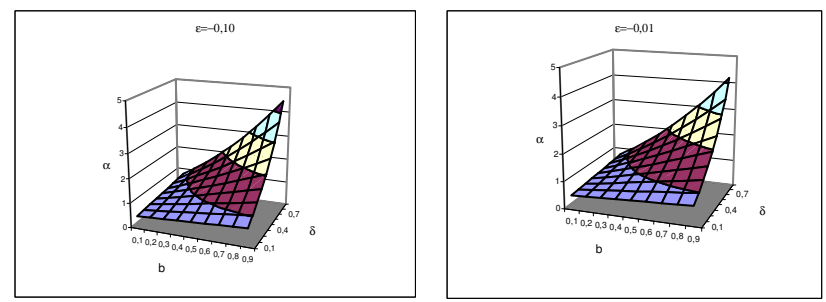

S'il apparaît que les dépenses publiques augmentent avec l'ampleur du choc d'offre négatif, on observe toutefois que c'est la progression conjointe de b et $\delta$ qui est déterminante pour l'évolution de $\alpha$. Par exemple, même si la préférence des autorités pour la production est très forte, le fait que l'impact des dépenses publiques sur la production soit faible peut conduire à une baisse des dépenses publiques. Les autorités intègrent ainsi le fait que l'utilisation de la variable budgétaire est ici de faible portée. Il semblerait que la crédibilité du policy-mix dépende de manière déterminante de $\delta$. Si ce dernier est très faible, alors il en va de même de la capacité des autorités à stabiliser un choc négatif par la politique budgétaire.

Les figures 2 et 3 analysent respectivement l'évolution des dépenses publiques et de l'inflation en fonction des chocs d'offre dans le régime de double règle flexible. Relativement à la figure 1, il apparaît que la progression des dépenses publiques est significativement plus faible. En outre, cet effet se renforce au fur et à mesure que l'ampleur des chocs d'offre négatifs augmentent. Autrement dit, dans une telle situation, les autorités ont bien tendance à absorber le choc par l'inflation. Cette dernière semble pour sa part peu sensible à l'impact des dépenses publiques sur la production. Elle est en fait très sensible à la préférence des autorités pour la production : la première augmente avec la seconde. Cependant, il apparaît un pallier qui semble traduire le fait que les autorités ne sont pas disposées à accepter un glissement inflationniste trop important. La règle budgétaire souple s'accompagne donc d'une dépréciation de faible ampleur: il est optimal de tolérer une telle dépréciation car elle permet l'absorption partielle du choc. Toutefois, la dépréciation 
monétaire est plus forte qu'en l'absence de règle budgétaire. Ce résultat est contre intuitif du point de vue des vertus disciplinantes des règles. Un tel résultat peut être expliqué par le fait que la présence de la règle budgétaire incite les autorités à un excès de vertu budgétaire. En renonçant à accroître les dépenses budgétaires, elles sont contraintes d'augmenter l'inflation afin de sauvegarder l'objectif de production.

Figure 2
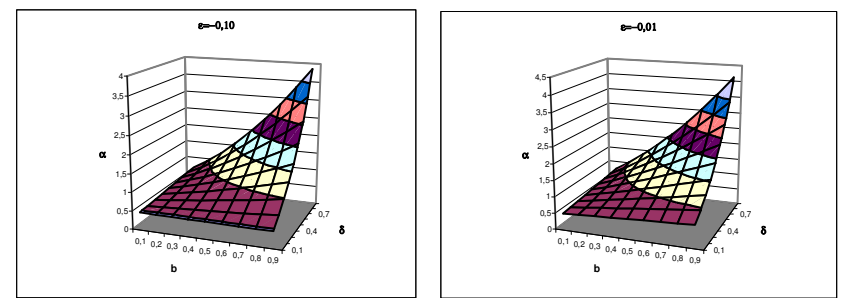

Figure 3
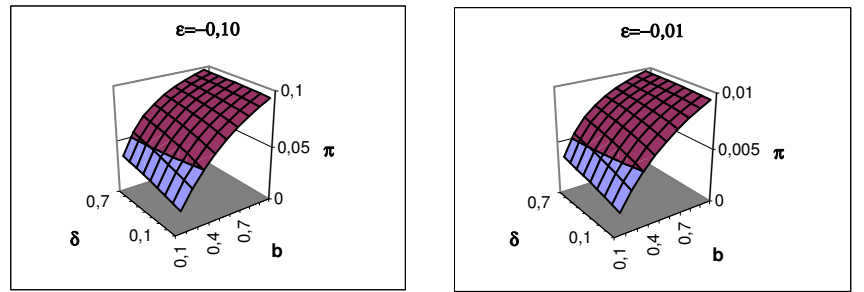

\section{CONCLUSION}

L'objectif du papier était de montrer que la défense du taux de change ne peut se concevoir sans considérer la crédibilité de l'ensemble du policy-mix. L'un des résultats essentiels est que la politique budgétaire peut exercer une action sur la pérennité d'une règle de change, dans la mesure où l'utilisation des marges de manœuvre budgétaire permet de relâcher la pression des anticipations sur le taux de change. La non utilisation de ces marges, au lieu de convaincre les agents de la détermination des autorités à défendre le taux de change peut conduire précisément à une augmentation des anticipations de dévaluation. Dans les pays qui n'ont pas de discipline budgétaire (Amérique Latine notamment), il peut être nécessaire pour renforcer la crédibilité de l'action sur le taux de change de mettre en œuvre des règles budgétaire du type Pacte de Stabilité et de Croissance. En effet, de telles règles budgétaires participent à la construction de la crédibilité du policy-mix avant la crise de change. Lorsque celles-ci se produisent, l'ampleur du choc est telle que le renforcement de la rigueur budgétaire est peu crédible. Ceci suggère que les autorités doivent se réclamer d'une clause de sortie dans le domaine budgétaire. 


\section{RÉFÉRENCES BIBLIOGRAPHIQUES}

AGÉNOR P.R. \& MASSON P.R. [1999], «Credibility, reputation, and the Mexican Peso crisis », Journal of Money, Credit, and Banking, vol.31, $\mathrm{n}^{\circ} 1$, p.70-84.

ARTUS P. [1995], «Effets internes et internationaux de l'indépendance des banques centrales », Revue Economique, vol.46, n³, p.857-867.

BAsurto G. \& GHosh A. [2000], «The interest rate - exchange rate nexus in the Asian crisis countries », IMF Working Paper, WP/00/19, February.

BENSAÏD B. \& JEANNE O. [1997], «The instability of fixed exchange-rate systems when raising the nominal interest rate is costly », European Economie Review, vol.41, p.1461-1478.

DE GRAUWE P. [1997], «Exchange rate arrangements between the ins and the outs », in P.R. MASSON, T.H. KRUEGER \& B.G. TURTELBOOM (eds), EMU and the international monetary system, IMF, Washington DC, p.97-118.

DRAZEN A. \& MASSON P.R. [1994], «Credibility of policies versus credibility of policymakers », Quarterly Journal of Economics, vol.109, n`3, p.735-754.

FURMAN J. \& STIGLITZ J.E. [1998], «Economic crises : evidence and insights from east Asia », Brookings Paper on Economic Activity, n², p.1-114.

GAVIN M. \& HAUSMANN R. [1998], « Preventing crisis and contagion : the domestic policy agenda », Communication à la conférence Crisis and contagion in emerging financial-markets: the new policy agenda, Inter-American Development Bank, October.

GOLDFAJN I. \& GUPTA P. [1999], «Does monetary policy stabilize the exchange rate following a currency crisis ? », IMF Working Paper, WP/99/42, March.

KAMINSKY G.L., LIZONDO S. \& REINHART C.M. [1997], «Leading indicators of currency crises », IMF Working Paper, WP/97/79, July.

KRUGMAN P.R. [1979], «A model of balance-of-payments crises », Journal of Money, Credit, and Banking, vol.11, $\mathrm{n}^{\circ} \gg$, p.311-325.

Moreno R., PAsadilla G. \& Remolona E. [1998], «Asia's financial crisis : lessons and policy responses », Pacific Basin Working Paper Series, PB 98-02, July.

OBSTFELD M. [1994], « The logic of currency crises », Cahiers Economiques et Monétaires, $\mathrm{n}^{\circ} 43, \mathrm{p} .189-213$.

RADELET S. \& SACHS J. [1998], «The onset of the East Asian financial crisis », Harvard Institute for International Development, Working Paper.

SACHS J. [1999], « Self-inflicted wounds », Financial Times, January 22.

SACHS J., TORNEll A. \& VelasCO A. [1996], «Financial crises in emerging markets : the lessons from 1995 », Brookings Papers on Economic Activity, n 1 , p.147-215. 
ANNEXE 1 : Principaux indicateurs économiques des pays sous programme du FMI.

\begin{tabular}{|c|c|c|c|c|}
\hline & $\mathrm{t}-1$ & $\mathrm{t}$ & $\mathrm{t}+1$ & $\mathrm{t}+2$ \\
\hline \multicolumn{5}{|c|}{ Taux de change (données trimestrielles) } \\
\hline Mexique (1995-1) & 5,32 & 6,82 & 6,31 & 6,42 \\
\hline Argentine (1995-1) & 0,99 & 1,00 & 1,00 & 1,00 \\
\hline Brésil (1997-3) & 1,08 & 1,10 & 1,12 & 1,14 \\
\hline Brésil (1999-1) & 1,21 & 1,72 & 1,77 & 1,92 \\
\hline Indonésie (1998-1) & 4650 & 8325 & 14900 & 10700 \\
\hline Corée du Sud (1997-4) & 915 & 1695 & 1383 & 1373 \\
\hline Thaïlande (1997-3) & 27,16 & 36,52 & 47,25 & 38,80 \\
\hline Thaillande (1998-1) & 47,25 & 38,80 & 42,31 & 39,31 \\
\hline Russie (1998-3) & 6,11 & 6,20 & 16,06 & 20,65 \\
\hline \multicolumn{5}{|c|}{ Taux d'intérêt à CT (données trimestrielles) } \\
\hline Mexique (1995-1) & 19,8 & 89,5 & 65,1 & 38,7 \\
\hline Argentine (1995-1) & 9,7 & 15,2 & 9 & 6,8 \\
\hline Brésil (1997-3) & 20,9 & 35,8 & 33,3 & 22,9 \\
\hline Brésil (1999-1) & 37,1 & 37,8 & 28,4 & 18,1 (oct.99) \\
\hline Indonésie (1998-1) & 41 & 58 & 66 & 74 \\
\hline Corée du Sud (1997-4) & 12 & 16 & 24 & 19 \\
\hline Thaillande (1997-3) & 12 & 19,3 & 20,1 & 20,6 \\
\hline Thaïlande (1998-1) & 20,1 & 20,6 & 18 & 9,6 \\
\hline Russie (1998-3) & 44,4 & 81,3 & 49,8 & 23,1 \\
\hline \multicolumn{5}{|c|}{ Production* / PIB $* * 1$ (données trimestrielles) } \\
\hline Mexique* (1995-1) & 114 & 106 & 101 & 101 \\
\hline Argentine* $(1995-1)$ & 139 & 121 & 121 & 128 \\
\hline Brésil* (1997-3) & 106,1 & 106,7 & 104,2 & 103,3 \\
\hline Brésil* (1999-1) & 100,0 & 100,5 & 101,7 & 101,4 \\
\hline Indonésie* (1998-1) & 97,6 & 95,7 & 96,5 & 97,9 \\
\hline Corée du Sud** (1997-4) & 112,4 & 122,8 & 98,2 & 103,3 \\
\hline Thaïlande** (1997-3) & 103,2 & 103,6 & 105,1 & 96,5 \\
\hline Thaillande** (1998-1) & 105,1 & 96,5 & 90,5 & 90,6 \\
\hline Russie** (1998-3) & 99 & 91,9 & 91,8 & 97,3 \\
\hline \multicolumn{5}{|c|}{ Soldes budgétaires (données annuelles) } \\
\hline Mexique (1995-1) & $0,7^{2}$ & $-0,9$ & $-0,4$ & $-1,3$ \\
\hline Argentine (1995-1) & $-0,2^{2}$ & $-3,4$ & $-3,2$ & $-1,9$ \\
\hline Brésil (1997-3) & $-5,9$ & $-5,9$ & -8 & -9 \\
\hline Brésil (1999-1) & -8 & -9 & n.d. & n.d. \\
\hline Indonésie (1998-1) & 1,3 & 1,4 & $-1,2$ & $-3,6$ \\
\hline Corée du Sud (1997-4) & 0,1 & $-1,4$ & $-4,4$ & $-4,9$ \\
\hline Thaillande (1997-3) & 2,4 & $-0,9$ & $-2,4$ & $-2,9$ \\
\hline Thaïlande (1998-1) & $-0,9$ & $-2,4$ & $-2,9$ & n.d. \\
\hline Russie (1998-3) & $-7,0$ & $-5,1$ & $-5,1$ (proj) & n.d. \\
\hline
\end{tabular}

${ }^{1}$ Mexique $(100=1990)$, Argentine $(100=1985)$; Brésil, Indonésie, Corée du Sud et Thaïlande $(100=1995)$, Russie (en \% de la même période de l'année précédente, source : Ministère des Finances de la Fédération de Russie). ${ }^{2} 1993$

Source : IMF, International Financial Statistics, various issues, Washington DC. 